\title{
Role of ectodermal derivatives as a personal identification tool-a forensic perspective
}

\begin{abstract}
Background of the study: The surface ectoderm, which gives rise to outer surface body tissues like epidermis, hair, and nails plays an important role in understanding the genetics, diseases and evolution of humans since decades. Various authors established characteristics occurrence of dermal and enamel configuration to find age, sex, blood groups and pathological conditions.
\end{abstract}

Aim: The present study was an attempt to analyze and determine the correlation between finger patterns and enamel patterns for personal identification in the field of forensics.

Methods and materials: The study comprised of thirty individuals' teeth extracted for orthodontic purposes or periodontal conditions irrespective of type and site of the tooth, age and sex. The digital image of both enamel and Left-thumb impression patterns obtained from the same individual were subjected to biometric conversion using Verifinger SDK 6.0 to analyze the morphological characteristics by automated finger pattern identification software.

Results: From the digital images of the two patterns analyzed it was found that 6 out of 30 samples showed similar but not exact identical patterned features to each other. The minutiae scores obtained for each enamel and finger pattern subjected to Pearson correlation statistical analysis showed no significant differences.

Conclusion: Similarities though not exact identical in the patterns observed in the present study signifies the conclusive association in the formation of enamel and finger pattern in an individual. Further research on a larger scale can aid in using this method as a key process in validation of a person in the field of forensics.

Keywords: biometric analysis, forensic science, thumb print, tooth print, verifinger
Volume 6 Issue 3 - 2018

Keerthi Narayan V,Varsha Vimal Kumar, Girish Hemadala, Sanjay Murgod, Sai Kavya M

Department of Oral Pathology and Microbiology, India

Correspondence: Keerthi Narayan V, Department of Oral Pathology and Microbiology, Rajarajeswari Dental College and Hospital, No: 14, Ramohalli Cross, Kumbalagodu, Bangalore, Karnataka-560074, India, Tel +9| 9|48326367, +9| 98948|8284, Email narayankrth55@gmail.com

Received: April 27, 2018 | Published: May 31 2018

\section{Introduction}

Identification of an individual by using diverse characteristics is a never ending challenging task. Dentist have a significant role in recognition process as oral cavity constitutes major evidence sites like lip, palate, teeth which has unique morphological features that differentiates from one person from another. ${ }^{1}$ Though several personal identification methods like chelioscopy, rugoscopy, bite mark analysis, DNA sampling, photography are greatly substantial in the recent years, an older yet highly significant individualistic method which plays a major part in personal identification either in the crime investigation or civil negotiations are Finger-patterns. Finger-patterns represent a set of minute raised ridges appearing as epidermal configuration on the volar aspect of the palmar and plantar regions that are highly constant and unique for each individual. ${ }^{2}$ Oral soft tissue examination for identification fails to reproduce details in accuracy and may not be an efficient method in cases of decomposed, burnt or buried bodies. In such scenario dental hard structures chiefly enamel being patterned hard tissue due to arrangement of enamel rod ends resist high decomposition and heat, found to be unique for every individual provides a key proof of identity. ${ }^{3}$

\section{Aim}

With the aim of analyzing the enamel rod end pattern on the tooth surface and finger pattern, the present study was performed to determine the correlation between finger patterns and enamel patterns for personal identification and also to check the reliability of this method to aid in recognition process in the field of forensics.

\section{Materials and methods}

The study comprised of 30 individuals' teeth extracted only for orthodontic purposes or periodontal conditions irrespective of type and site of the tooth, age and sex followed by recording their left thumb impressions after obtaining consent.

\section{Technique for recording enamel pattern}

The tooth to be analyzed was scaled and polished. Generally, the middle third of the facial surface of the tooth is selected for analysis as it is most resistant to changes due to attrition, abrasion, etc. The surface was etched with $37 \%$ ortho-phosphoric acid for 20 seconds, washed with water and dried. A thin layer of acetone was then applied over a small piece of cellulose acetate film and placed immediately over the etched surface of the tooth without any finger pressure for 20 minutes. The acetone dissolves a layer of cellulose acetate and the dissolute settles down along the irregularities on the enamel surface (Figure 1). The film was gently peeled after 20 minutes and transferred onto a clean glass slide. The slides were then observed under light microscope attached with a digital camera. The digital image of a suitable area was obtained under 40X (Figure 2). This obtained digital image was initially subjected to biometric conversion by Verifinger standard SDK 6.0 version where the software recognizes the different enamel rod endings as series of lines organized in different directions. 
The software uses certain points called as minutiae for identification which are breaks of lines, line endings, dots and/or empty spaces between the two lines (Figure 3). The biometric patterns obtained were evaluated for morphological characteristics by automated finger identification system.



Figure I Application of acetone and small piece of cellulose acetate film over the etched surface.

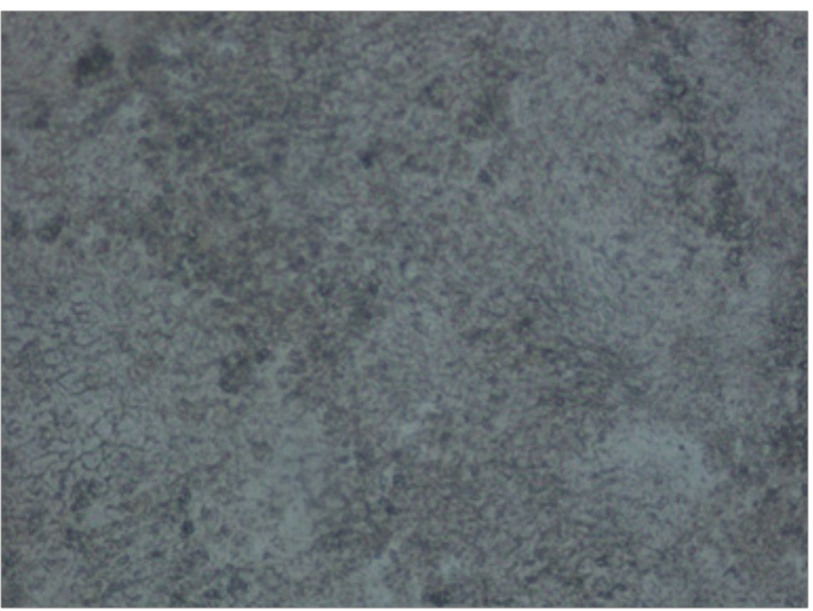

Figure 2The enamel rod endings observed using light microscope under 40X magnification.

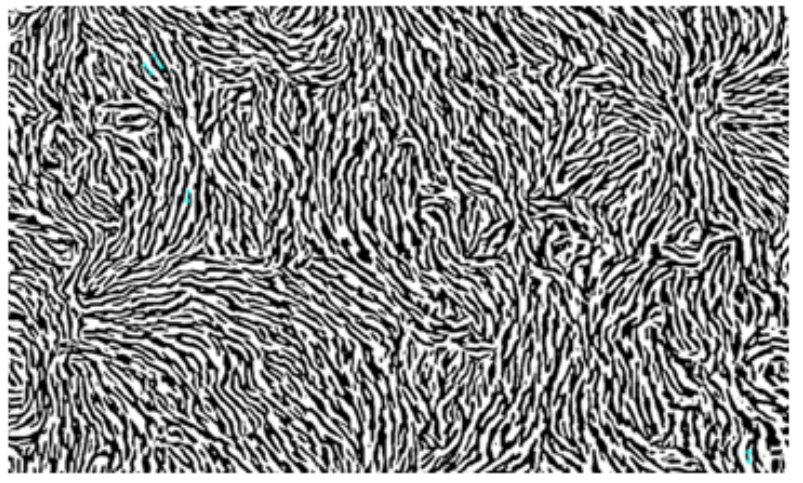

Figure 3 Biometric patterned image of enamel rod endings. (Verifinger standard SDK Version 6.0 software).

\section{Technique for recording finger pattern}

Left thumb finger was cleaned and was pressed touching the blue ink stamp pad with gentle pressure for 20-40seconds. The finger was slowly raised and placed immediately on a clean white paper without any pressure. The patterns were examined for morphological patterns visually to check for flaws and was analyzed for pattern characteristics as classified by the science of finger-patterns-classification and uses, by the Federal Bureau of Investigation (FBI) identification division 1957. ${ }^{4}$ The digital image obtained was subjected to biometric conversion by Verifinger standard SDK 6.0 version for recording the minutiae scores (Figure 4). The biometric patterned image for each pattern was obtained and was subjected to evaluation of pattern characteristics by automated finger identification system. Enamel patterns and finger patterns obtained from the same individuals were analyzed for pattern characteristics using Automated Finger-pattern Identification System (AFIS) to determine the similarity between the two patterns (Figure 5). The minutiae scores recorded by Verifinger standard SDK 6.0 version was subjected to statistical analysis to check the correlation between the two patterns and also to check the reliability of the digital analysis method (Figure 6).

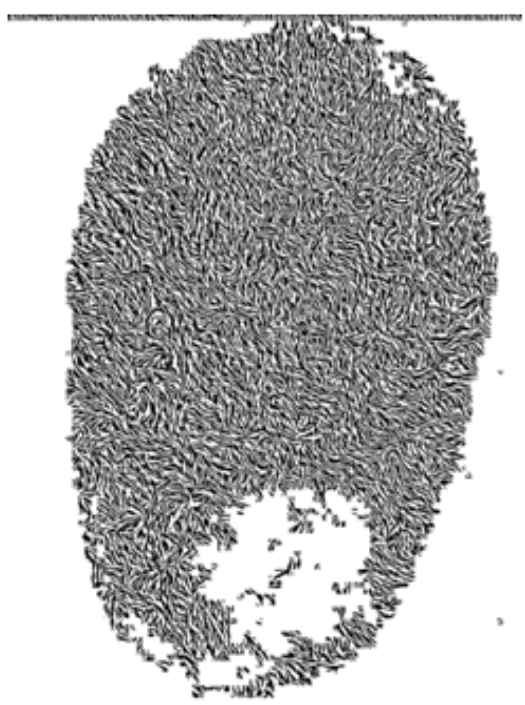

Figure 4 Biometric patterned image of thumb finger pattern. (Verifinger standard SDK Version 6.0 software).

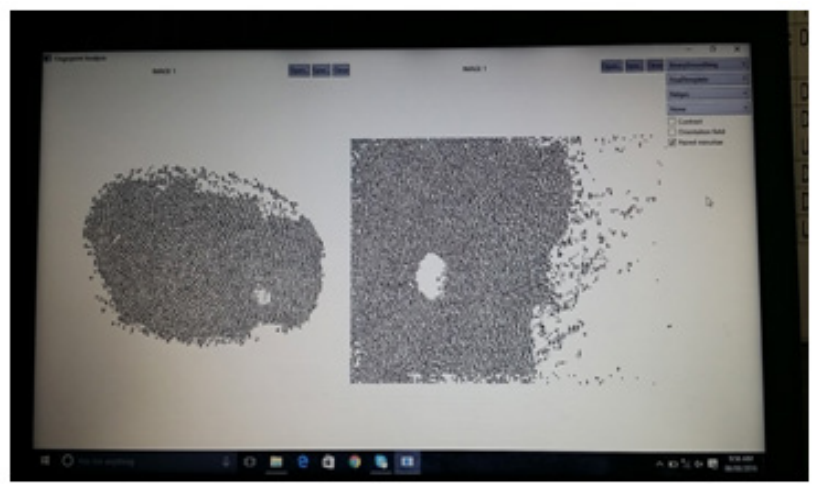

Figure 5 Biometric patterned image analyzed by automated finger identification system software. 


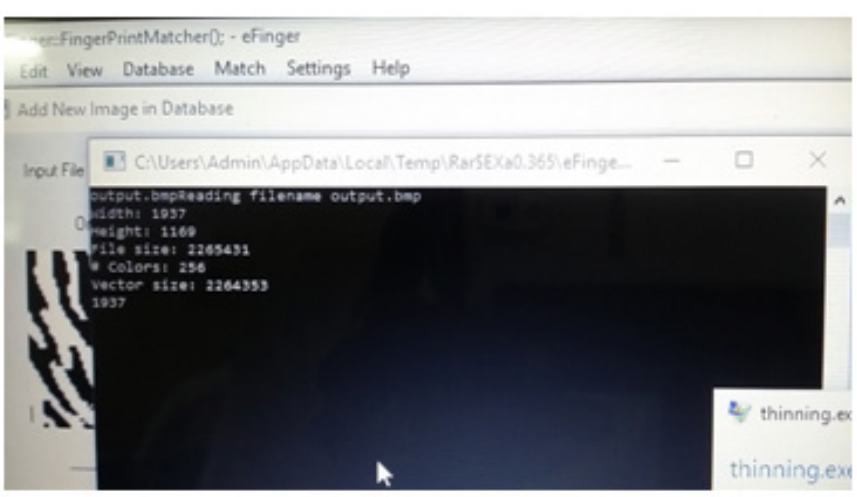

Figure 6 Minutiae scores obtained for both enamel and finger pattern analyzed.

\section{Statistical analysis}

Statistical analysis was performed with the help of SPSS version 21.0. Percentage of distribution of various patterns of both enamel and finger patterns among the study samples were tabulated. Comparison of morphological characteristics between the two patterns was performed and significance was checked by using chi square goodness of fit test. Correlation and reliability between the two patterns recorded as minutiae scores were compared by using Pearson correlation statistics to determine the relationship between them.

\section{Interpretations}

Distribution of different finger pattern among the study samples shows loop pattern in 4 cases (13.3\%) and its variations like radial loop in 4 cases (13.3\%), ulnar loop in 7 cases $(23.3 \%)$ showing the overall predominance of loop pattern and its variants (49\%). Whorl pattern was seen in 5 cases and plain whorl pattern in 7 cases $(23.3 \%)$ and least being the arch pattern in only 3 cases $(10.1 \%)$ (Table 1$)$. In case of enamel pattern about 8 various patterns are obtained. Loop pattern in 11 cases $(36.7 \%)$ and its variation radial loop in 1 case showing the overall predominance of loop pattern and its variants (40\%). Whorl pattern of closed type is seen in 6 cases and open pattern in 6 cases comprising a total of 12 cases (40\%) and least being the arch pattern including branched type in only 3 cases (10.1\%) (Table 2 ). Comparison of the morphological pattern characteristics between finger pattern and enamel pattern showed among the 30 samples obtained for the study only 6 samples showed similar patterned features to each other and 24 samples were not matching. Statistical insignificance was obtained (Table 3). The obtained minutiae scores of both enamel and finger-patterns were tabulated. However Correlation between the Finger pattern $\&$ enamel pattern minutiae scores analyzed by using Pearson correlation statistics showed 0.27 positive correlation with no significant differences (Table 4).

Table I Distribution of various finger patterns among the individuals

\begin{tabular}{lll}
\multicolumn{3}{l}{ Distribution of various finger pattern among the individuals } \\
\hline Finger pattern & $\mathbf{n}$ & $\%$ \\
\hline Arch & 3 & $10.00 \%$ \\
Loop & 4 & $13.30 \%$ \\
Plain Whorl & 7 & $23.30 \%$ \\
Radial loop & 4 & $13.30 \%$ \\
Ulnar loop & 7 & $23.30 \%$ \\
Whorl & 5 & $16.70 \%$ \\
\hline
\end{tabular}

Table 2 Distribution of various enamel pattern among the study individuals

\begin{tabular}{lll}
\hline Enamel pattern & N & $\%$ \\
\hline Arch & 2 & $6.70 \%$ \\
Arch branched & I & $3.30 \%$ \\
Linear branched & I & $3.30 \%$ \\
Loop & II & $36.70 \%$ \\
Radial loop & I & $3.30 \%$ \\
Wavy unbranched & 2 & $6.70 \%$ \\
Whorl closed & 6 & $20.00 \%$ \\
Whorl Open & 6 & $20.00 \%$ \\
\hline
\end{tabular}

Table 3 Comparison of the morphological profile matching using chi square goodness of fit test

\begin{tabular}{|c|c|c|c|c|}
\hline \multicolumn{5}{|c|}{$\begin{array}{l}\text { Comparison of the morphological profile between finger pattern } \\
\text { and enamel pattern matching using Chi square goodness of fit } \\
\text { test }\end{array}$} \\
\hline Morphological profile & $\mathbf{N}$ & $\%$ & $c^{2}$ Value & P-Value \\
\hline Matching & 6 & $20 \%$ & \multirow{2}{*}{2.4} & \multirow{2}{*}{$0.548^{*}$} \\
\hline Not Matching & 24 & $80 \%$ & & \\
\hline
\end{tabular}

Table 4 Correlation between the finger pattern (FP) \& enamel pattern (EP) minutiae scores using Pearson correlation statistics

\begin{tabular}{llll}
\multicolumn{3}{l}{$\begin{array}{l}\text { Correlation between the Finger pattern\& enamel pattern } \\
\text { minutiae scores using Pearson correlation statistics }\end{array}$} \\
\hline Variables & Values & $\begin{array}{l}\text { FP_minutiae } \\
\text { scores }\end{array}$ & $\begin{array}{l}\text { EP_minutiae } \\
\text { scores }\end{array}$ \\
\hline \multirow{4}{*}{ FP_minutiae scores } & $\mathrm{R}$ & $\mathrm{I}$ & 0.27 \\
& $\mathrm{P}$ & & 0.16 \\
& $\mathrm{~N}$ & 30 & 30 \\
EP_minutiae scores & $\mathrm{R}$ & 0.27 & $\mathrm{I}$ \\
& $\mathrm{P}$ & 0.16 & \\
& $\mathrm{~N}$ & 30 & 30 \\
\hline
\end{tabular}

\section{Discussion}

The study of various patterned surfaces formed by naturally occurring ridges on palms, fingers and soles was in practice over many decades. Among the patterned surfaces finger patterns were unique for each individual and dental professional considered these as a highly sensitive indicator of numerous intrauterine dental anomalies and to evaluate various pathological conditions like dental caries, malocclusion, periodontal diseases, oral submucous fibrosis and congenital conditions like cleft lip, cleft palate and also in the field of forensic science for personal identification. Galton in 1892 classified the finger patterns into three major groups as loops, arches and whorls. ${ }^{5}$ In the present study FBI identification classification of finger patterns proposed in 1957 which sub classifies the major pattern as Arch (plain, tented), Loop (ulnar, radial, double loop), whorl (plain, accidental) was followed. In humans the mammary buds began to mature during the $6^{\text {th }}$ week of intrauterine life as a descending growth of epidermis into the underlying ectomesenchyme. This series of events from initiation till formation of ridges lasts till $12^{\text {th }}-13^{\text {th }}$ week. The formation of the epidermal ridges was determined predominantly by genetic factors though host and environmental factors has a significant role in the formation process. ${ }^{6}$ The epidermal ridges of the 
fingers, palms as well as facial structures like lip, alveolus, tooth bud were also derived from the ectodermal layer during $6^{\text {th }}-7^{\text {th }}$ week of intrauterine life. Any significant alterations or disturbances during this period have a direct effect on all these structures. ${ }^{7}$

Dutta et al., ${ }^{8}$ in a comparative study on finger patterns in patients with oral submucosis fibrosis concluded palmer dermatoglyphics can predict the occurrence of OSMF and also can diagnose a person in the prefibrosis stage. ${ }^{8}$ Abhilash et al., ${ }^{9}$ in a case control study on 1250 children in the age group of 5 to 12 years observed caries susceptibility of an individual was higher with incidence of whorl pattern and was the least with loop pattern. ${ }^{9}$ In the recent years with the advancing techniques various authors have proposed several methods like Finger patterns, lip patterns, rugae patterns, DNA analysis, and photographs for personal identification. Among these Finger patterns were considered as one of the vital method of identification in both civil and criminal cases in the field of forensic science in the recent years. Adamu et al., ${ }^{2}$ conducted a study on 820 subjects found a positive relationship between lip pattern, finger pattern and observed the most common finger pattern were loops (54.8\%) followed by whorls (39.9\%) and arches $(5.3 \%)$ among both the sexes. ${ }^{2}$ The present study exhibited similar results where loop pattern showed predominance of $49.9 \%$ (loop, radial, ulnar) followed by whorl (40.1\%) and least being the arch pattern (10.1\%). Similar study by Naik et al., ${ }^{1}$ on 100 students concluded a significant correlation between lip-pattern and finger pattern as a tool for gender identification. ${ }^{1}$ Patil et al., ${ }^{10}$ proposed a method of estimating age of the person and identification through their finger patterns images. ${ }^{10}$

These human identification methods commonly fail to predict accurate information if the bodies were decomposed, severely traumatized or burnt. When soft tissue cannot provide a reliable information, identification is possible with skeletal remains especially teeth. Teeth can withstand high temperature, forces and resist postmortem decomposition. ${ }^{12}$ The formation is genetically controlled. ${ }^{12}$ Similar to the dermal pattern, the ectoderm derived cells ameloblasts lay down the enamel rods in an unorganized inter twinning path. This is replicated on the outer surfaces of the enamel as patterns of the ends of series of adjacent enamel rods. These enamel rod end patterns or enamel patterns can be recorded by using cellulose acetate strip, cellophane tape or rubber base impression material. ${ }^{13}$ Manjunath et al. ${ }^{13}$ in his preliminary study for recording enamel rod end patterns established Cellulose acetate film peel as a reliable material for recording rod endings and reported similar to the patterns obtained from the thumb finger, enamel pattern also expressed distinctive pattern for every individual. He identified 8 distinct sub patterns namely wavy-branched, wavy-unbranched, linear-branched, linear-unbranched, whorl-open, whorl closed, loop and stem-like. ${ }^{14}$ Correspondingly in the present study all the eight types were identified but loop pattern showed higher predominance of 11 in 30 samples (36.7\%) and least being arch branched, linear branched, radial loop which showed only 1 out of 30 samples in each type respectively $(3.3 \%)$.

Gupta et al., ${ }^{15}$ in a study observed that enamel patterns were composed of varied patterns and sub-patterns and found that the patterns were completely dissimilar from one another when related between teeth of the same individual and also for diverse group of people. ${ }^{15}$ Joshi PS et al., ${ }^{3}$ in a study also observed inter and intra individual dissimilarities between the enamel patterns obtained. ${ }^{3}$ The present study showed loop pattern being the most common among both the enamel and finger pattern and arch pattern being the least. Manjunath et al., ${ }^{16}$ in a study established acetate peel technique with Verifinger standard SDK as a reliable technique in analyzing enamel rod end patterns and found that Verifinger software was able to identify the patterns recorded and comparison of the minutiae scores showed no significant differences as the software uses the distribution and association of these minutiae points to each other in a particular area. ${ }^{16}$ Similarly in the present study no differences were observed between minutiae scores of finger pattern and enamel pattern recorded by the software. A positive pattern characteristics correlation was found between the two patterns analyzed. The correlation found between the enamel pattern and finger pattern for personal identification was not statistically significant. In the present study though higher level of significance was not obtained, owing to the positive correlation between the patterns this method may create an opportunity to use the correlation between the finger pattern and enamel pattern.

\section{Limitations}

Despite being the hardest mineralized substance in human body, the enamel surface is usually subjected to vary by various environmental factors. Any alterations in the morphology due to physical, mechanical or chemical factors can impede the identification process. Further study on analysis of enamel rods at different levels can give more accurate results along with the use of automated biometrics.

\section{Conclusion}

Application of finger pattern as an identification of individual in circumstances like civil or criminal is highly considered owing to its uniqueness. Likewise in condition where the soft tissue analysis is not possible such as mass disasters or fire accidents hard tissue counterpart are the only sources for identification process. The enamel patterns though unique to an individual its reproducibility and permanency have to be determined in conjugation with other identification methods. In the present study though higher level of significance was not obtained, owing to the positive correlation between the patterns this method may create an opportunity to use the correlation between the finger pattern and enamel pattern as an additional tool in identification process when a very few evidences are available. The significance of this method as a personal identification tool can be more accurately determined only by evaluating in a larger scale.

\section{Acknowledgements}

None.

\section{Conflict of interest}

Author declares that there is no conflict of interest.

\section{References}

1. Naik R, Ahmed Mujib BR, Telagi N, et al. Comparative analysis of lip with thumb patterns; an identification tool in personal authentication. $J$ Oral Maxillofac Pathol. 2017;21(1):171-5.

2. Adamu LH, Taura MG, Hamman WO, et al. Relationship of thumb patterns and lip patterns among Nigerians. IOSR Journal of Dental and Medical Sciences. 2013;9(2):12-17.

3. Joshi PS, Bhosale SS. Study of Enamel Rod End Patterns Using Acetate Peel Technique and Automated Biometrics for Personal Identification. Int J Dent Med Res. 2014;1(4):47-50. 
4. Prabha LJ, Thenmozhi R. A Short Review On dermatoglyphics. J Pharm Sci \& Res. 2014;6(4):200-202.

5. Ramani P, Abhilash PR, Herald JS, et al. Conventional DermatoglyphicsReviewed Concept: A Review. International Journal of Pharma and Bio Sciences. 2011;2(3):446-458.

6. Ceena Denny E, Junaid A, Nandita S, et al. Dermatoglyphics in Dentistry -A Review. Int J Cur Res Rev. 2013;5(21):30-33.

7. Madhusudan K, Hiren PP, Umesh K, et al. Relationship between Dermatoglyphics, Chiloscopy and Dental Caries among Dental Students of Visnagar Town, Gujarat. International Journal of Advanced Research. 2015;3(7):952-959.

8. Dutta N, Shetty R, PandeyV. Comparison of finger pattern patterns in patients with and without oral submucous fibrosis- A dermatoglyphics study. International Journal of Contemporary Medical Research. 2016;3(4):1172-1173.

9. Abhilash PR, Divyashree R, Patil SG, et al. Dermatoglyphics in Patients with Dental Caries: A Study on 1250 Individuals. J Contemp Dent Pract. 2012;13(3):266-274

10. Patil BGV, Rafi M. Human Age Estimation through Finger Pattern. International Journal of Innovative Research in Computer and Communication Engineering. 2015;3(4);3530-3534.
11. Deshmukh RA. Need for Newer Techniques for Personal Identification. J Forensic Res. 2015;6(1):284-285

12. Bharanidharan R, Karthik R, Rameshkumar A, et al. AmeloglyphicsAn adjunctive aid in individual identification. SRM J Res Dent Sci. 2014;5(4):264-8.

13. Manjunath K, Sivapathasundharam B, Saraswathi TR. Efficacy of various materials in recording enamel rod endings on enamel surface for personal identification. J Forensic Dent Sci. 2011;3(2):71-76.

14. Manjunath K, Sriram G, Saraswathi TR, et al. Enamel rod end patterns: a preliminary study using acetate peel technique and automated biometrics. J Forensic Odontol. 2008;1(1):33-36.

15. Gupta N, Jadhav K, Ahmed Mujib BR, et al. Is Re-Creation Of Human Identity Using Tooth Patterns? An Experimental Study to Aid In Identification. Forensic Sci Int. 2009;192(1-3):67-71.

16. Manjunath K, TR Saraswathi, G Sriram, et al. Reliability of automated biometrics in the analysis of enamel rod end patterns. Journal of Forensic Dental Sciences. 2009;1(1):32-36. 